BULLETIN (New Series) OF THE

AMERICAN MATHEMATICAL SOCIETY

Volume 49, Number 4, October 2012, Pages 469-474

S 0273-0979(2012)01382-4

Article electronically published on July 3, 2012

\title{
INTRODUCTION TO THE PAPERS OF R. THOM AND J. MATHER
}

\author{
MARK GORESKY
}

\begin{abstract}
Immediately following the commentary below, the previously published article by R. Thom is printed in its entirety: R. Thom, Ensembles et morphismes stratifiés, Bulletin of the American Mathematical Society 75 (1969), no. 2, 240-284 (French). This is followed by the first publication of the 1970 lecture notes of J. Mather, Notes on topological stability.
\end{abstract}

For any closed set $E \subset \mathbb{R}^{n}$ there exists a $C^{\infty}$ function $f: \mathbb{R}^{n} \rightarrow \mathbb{R}$ such that $E=f^{-1}(0)$. This includes the Cantor set, the Sierpiński sponge, the Snowflake, and other sets of fractional Hausdorff dimension. How does one prove that this sort of behavior cannot happen when $f$ is an analytic function or an algebraic function? These questions were approached about 80 years ago when it was shown ([38, 15, 13, 16]) that algebraic sets could be triangulated 1$]$ For many years the 1932 paper 13] was cited as the only known proof that complex algebraic sets were locally contractible. But these papers are difficult to follow, and for decades the triangulability of algebraic and analytic sets was treated with some suspicion. Later articles, such as [30, 3, 18, and especially [10, 11, 8], finally put these questions to rest. However, the interesting structure of the singularities of an algebraic set is not easily2 described using simplices, and people began to look for a more intelligent way to decompose an algebraic set into (fewer, larger) pieces, starting with the nonsingular part.

Hassler Whitney struggled with these questions for decades. In 1946 he wrote Complexes of manifolds [40], in which he considered spaces that were glued together out of smooth manifolds much in the way that a cell complex is glued together from cells. In 1957 Whitney showed in 41] that it is possible, in any algebraic set, to choose an open dense nonsingular part such that its complement is an algebraic set of smaller dimension. Therefore this procedure can be repeated so as to give a finite filtration by closed subsets $X_{0} \subset X_{1} \subset \cdots \subset X_{n}$ such that $X_{r+1}$ is obtained from $X_{r}$ by attaching a (possibly empty) smooth manifold $S_{r+1}:=X_{r+1}-X_{r}$ of dimension $r+1$.

One might hope that the resulting decomposition into pieces $X=\coprod_{r} S_{r}$ is locally trivial - that any two sufficiently nearby points $x, y \in S_{r}$ should have neighborhoods that are isomorphic (in a sense to be made precise below) by an isomorphism (a homeomorphism, or perhaps, a diffeomorphism) that preserves the induced filtrations. In 1962 René Thom made a first attempt in 34 to make precise such a notion of a locally trivial stratification. He proposed that each stratum $S$ should have a

\footnotetext{
${ }^{1}$ In other words, every compact algebraic set is homeomorphic to a finite simplicial complex.
} 


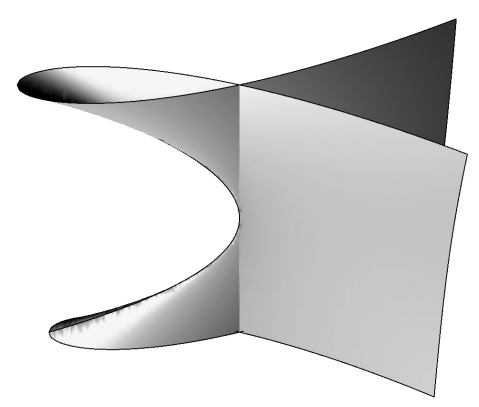

FIGURE 1

"good" tubular neighborhood $T_{S}$, together with a projection function $T_{S} \rightarrow S$ and a "carpeting" function $2 S \rightarrow \mathbb{R}_{>0}$, both of which should have maximal rank when restricted to any larger stratum.

It turns out that Whitney's procedure as described above is not enough to give such a locally trivial stratification. In a remarkable 1965 paper [42] Whitney described several important examples that illustrate the problems. The first example (see Figure 1) is a two-dimensional algebraic set with an obvious nonsingular part that appears to consist of several sheets of paper. If we throw away this twodimensional part, then what remains is a smooth one-dimensional manifold. But clearly there is one point on this singular stratum that is special. The problem is that we "threw away" the nonsingular part too soon, because the way that it twists around the singular stratum changes at the special point. It was necessary to find a way to identify that point as special, and Whitney [42, Sect. 8, p. 228] proposed his Conditions $\mathrm{A}$ and $\mathrm{B}$ as possible candidates 3 He also outlined a proof (with details in [43]) that complex analytic sets can be Whitney stratified (that is, stratified so as to satisfy Whitney's conditions A and B) with complex analytic strata. If we stratify the preceding example with two strata, then condition B fails at the origin, forcing us to (correctly) consider the origin as a third stratum.

Large classes of naturally occurring sets have since been shown to admit Whitney stratifications; see (for example) [19, 6, 5, 7, 9, 12, 14, 1, 31, 32. However, conditions $\mathrm{A}$ and $\mathrm{B}$ still allow for some dangerous pathologies. Thom points out in 35, that the spiral $r=\exp \left(-\theta^{2}\right)$ satisfies the Whitney conditions, but it turns infinitely many times as it approaches the origin.

An even more worrisome example is described in Whitney's article 42]. Typically, a singular space $X$ might arise as a subset of Euclidean space, or as a subset of a smooth manifold $M$. A reasonable goal is to ask for a stratification of $X$ so that if $x, y \in X$ lie in a single (connected) stratum, then there should exist open neighborhoods $U_{x}, U_{y} \subset M$ and a diffeomorphism $\phi: U_{x} \rightarrow U_{y}$ which takes $X \cap U_{x}$ to $X \cap U_{y}$ in a stratum-preserving way. It turns out that this is too much to hope for, even if $X$ is a complex algebraic set. Whitney's example is shown in Figure 2 , any smooth isotopy that flows along the $x$-axis will have the property that, when restricted to a normal slice through that stratum, the derivative will map four lines

\footnotetext{
${ }^{2}$ In [26], Mather replaced this with the more intuitive and useful notion of a function that measures the "distance" from the stratum $S$.

${ }^{3}$ In [26, Mather showed that condition B implies condition A.
} 


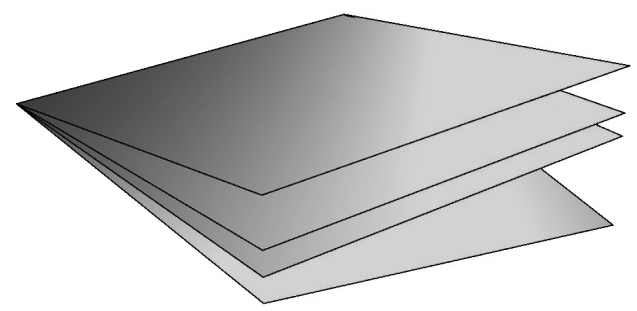

Figure 2

in $\mathbb{R}^{2}$ to four lines in $\mathbb{R}^{2}$, three of which do not move but the fourth does. However the derivative is determined by the fact that the first three lines do not move; therefore, it cannot move the fourth line either.

Thus, the best we can hope for is that a stratification should be topologically locally trivial: given points $x, x^{\prime}$ in a connected component of a single stratum, there should be a continuous map (which is smooth on each stratum, but not necessarily differentiable on the ambient manifold) which maps a neighborhood of $x$ homeomorphically, in a stratum-preserving way, to a neighborhood of $x^{\prime}$. But what hope is there to construct such a homeomorphism that is continuous, stratum preserving, smooth on each stratum, and yet is not differentiable, on an $n$-dimensional space that might be as pathological as Thom's spiral? Thom had the daring idea to address this problem by constructing a discontinuous vector field on the stratified space whose flow is continuous but not differentiable. The flow of such a controlled vector field is then the desired homeomorphism. In some sense, this is the key idea that separates a reasonable (i.e., a stratified) singular space from an unreasonable space (such as a Cantor set): the reasonable spaces have locally constant topological type.

Thom had in mind a particular application to the study of smooth mappings. Let $M$ and $N$ be smooth manifolds with $M$ compact. A smooth mapping $f: M \rightarrow N$ is said to be differentiably stable if, for every sufficiently nearby mapping $g: M \rightarrow N$, there exist diffeomorphisms $\phi: M \rightarrow M$ and $\psi: N \rightarrow N$ that convert $f$ into $g$; that is, $g=\psi^{-1} \circ f \circ \phi$. One might guess that such differentiably stable maps form an open and dense set in the space of all smooth mappings. For $\operatorname{dim}(N)=1$ this is a basic result in Morse theory, and for $\operatorname{dim}(M)=\operatorname{dim}(N)=2$ it follows from Whitney's 1955 article [39. But in 1960, Thom gave a counterexample [17, §11] when $\operatorname{dim}(M)=\operatorname{dim}(N)=16$.

The complete answer to this question was determined in a remarkable series of articles by John Mather 20, 21, 22, 23, 24, 25 in which he determined necessary and sufficient conditions, depending only on $\operatorname{dim}(M)$ and $\operatorname{dim}(N)$, for the differentiably stable mappings to form an open and dense set in the space of all smooth mappings. Those conditions are that the pair $(m=\operatorname{dim}(M), n=\operatorname{dim}(N))$ should satisfy any one of the following relations:

$$
\begin{aligned}
n-m & \geq 4 \text { and } m<\frac{6}{7} n+\frac{8}{7}, \\
3 \geq n-m & \geq 0 \text { and } m<\frac{6}{7} n+\frac{9}{7}, \\
n-m & =-1 \text { and } n<8 \\
n-m & =-2 \text { and } n<6 \\
n-m & =-3 \text { and } n<7 .
\end{aligned}
$$


It turns out that the difficulty is caused, in some sense, by the smoothness requirement on $\phi, \psi$, which we now relax. Two smooth mappings $f, g: M \rightarrow N$ are said to be topologically equivalent if there exist homeomorphisms $\phi, \psi$ such that $g=\psi^{-1} \circ f \circ \phi$. A smooth mapping $f: M \rightarrow N$ is said to be topologically stable if every sufficiently nearby mapping $g$ is topologically equivalent to $f$. Thom conjectured that for any two smooth manifolds $M, N$ (with $M$ compact), the set of topologically stable mappings forms an open and dense subset of $C^{\infty}(M, N)$.

In a series of papers that spanned a decade (including [34, 33, 35]), Thom proposed the whole apparatus of stratification theory and controlled vector fields in order to establish this result. His most detailed treatment of stratification theory appears in the paper, Ensembles et morphismes stratifiés [35, which is reprinted here. Unfortunately, Thom's treatment does not provide a complete proof of the density of topologically stable mappings 4 Moreover, his proof that Whitney stratifications are topologically locally trivial is extremely difficult to understand and lacks essential details. Thom's outline is geometric and full of wonderful ideas, but it is written in a way that gives the reader very little guidance on how to start filling in the gaps.

In 1970 John Mather worked out a proof of Thom's conjecture (that the set of topologically stable mappings is open and dense in the space $\left.C^{\infty}(M, N)\right)$, and he began the task of writing a book that would explain his proof. In 1970 Mather gave a series of lectures on this material at Harvard, and his notes (which were to be part of the first chapter of the book) were written up [26]. These notes are precise, clear and very readable; they place the theory of stratifications on a firm foundation. They contain proofs of the isotopy lemmas and of the fact that every Whitney stratification is locally trivial. The arguments involve delicate existence and extension theorems for tubular neighborhoods, combined with double inductions among the strata.

Although the later chapters of Mather's book were never completed, the Notes circulated widely in mimeographed and photocopied form for many years. They became the standard source for the foundations of stratification theory, and they have often been summarized and paraphrased (for example, in [4, Chapter 2], 37, Chapter 1], 29, Chapter 3]). They are being formally published here for the first time (with minor corrections by John Mather). The rest of the project to prove that topologically stable mappings are dense was outlined in [27] with details in [28; ; see also [4] and [2].

The two papers (re)printed here represent a turning point in the theory of stratifications. Their significance extends well beyond the study of the stability of smooth mappings. Indeed, stratification theory has become an indispensable tool in many branches of topology, geometry, algebra, combinatorics, and number theory, and the word stratification is now part of the standard vocabulary of modern mathematics. The subject itself continues to grow and evolve.

\section{ACKNOWLEDGMENTS}

The author is grateful to the referee for many useful comments on an earlier version of this note.

\footnotetext{
${ }^{4}$ Thom acknowledges this in [36, p. 204].
} 


\section{REFERENCES}

[1] L. van den Dries, Tame Topology and $\mathcal{O}$-minimal Structures, Cambridge Univ. Press, Cambridge, 1998. MR1633348 (99j:03001)

[2] A. du Plessis and T. Wall, The Geometry of Topological Stability, London Math. Soc. Monographs 9, Oxford University Press, Oxford, 1995. MR.1408432(97k:58024)

[3] B. Giesecke. Simpliziale Zerlegung abzählbarer analytische Räume, Math. Zeit. 83 (1964), 177-213. MR0159346 (28:2563)

[4] C. Gibson, K. Wirthmüller, A. du Plessis, and E. Looijenga, Topological Stability of Smooth Mappings, Lecture Notes in Mathematics 552, Springer Verlag, 1976. MR0436203 (55:9151)

[5] Slicing and intersection theory for chains associated with real analytic varieties, Acta Math. 129 (1972), 57-136. MR0315561 (47:4110)

[6] R. Hardt, Homology and images of semianalytic sets, Bull. Amer. Math. Soc. 80 (1974), 675-678. MR0348140(50:638)

[7] R. Hardt, Stratification of real analytic mappings and images, Invent. Math. 28 (1975), 193208. MR0372237 (51:8453)

[8] R. Hardt, Triangulation of subanalytic sets and proper light subanalytic maps, Invent. Math. 38 (1976/77), 207-217. MR0454051(56:12302)

[9] H. Hironaka, "Subanalytic sets", in Number Theory, Algebraic Geometry, and Commutative Algebra (Dedicated to Akizuki), Kinokunia, Tokyo Japan, 1973, 453-493. MR0377101 $(51: 13275)$

[10] H. Hironaka, "Triangulations of algebraic sets", in Algebraic Geometry (Proc. Sympos. Pure Math., 29, Amer. Math. Soc., Providence, Rhode Island, 1974, pp. 165-185. MR0374131 $(51: 10331)$

[11] H. Hironaka, Introduction to real-analytic sets and real-analytic maps. Quaderni dei Gruppi di Ricerca Matematica del Consiglio Nazionale delle Ricerche. Istituto Matematico "L. Tonelli" dell'Università di Pisa, Pisa, 1973. iii+162 pp. MR0477121 (57:16665)

[12] H. Hironaka, "Stratification and flatness", in Real and Complex Singularities, Proc. Ninth Nordic Summer School/NAVF Symp. Math. Oslo, Sijthoff and Noordhoff, Alphen aan den Rijn, Netherlands, 1977, 199-265. MR0499286 (58:17187)

[13] B. C. Koopman and A. B. Brown, On the covering of analytic loci by complexes, Trans. Amer. Math. Soc. 34 (1932), 231-251. MR1501636

[14] T. Le Loi, "Lecture 2: Stratifications in o-minimal structures" in The Japanese-Australian Workshop on Real and Complex Singularities-JARCS III, 31-39, Proc. Centre Math. Appl. Austral. Nat. Univ., 43, Austral. Nat. Univ., Canberra, 2010. MR2763234 (2012f:03059)

[15] S. Lefschetz, Topology, Amer. Math. Soc. Colloq. Publ., New York (1930).

[16] S. Lefschetz and J. H. C. Whitehead, On analytical complexes, Trans. Amer. Math. Soc. 35 (1933), 510-517. MR:1501698

[17] H. Levine, "Singularities of differentiable mappings, notes of lectures by R. Thom", University of Bonn, 1960; reprinted in Proceedings of Liverpool Singularities-Symposium I, Lecture Notes in Mathematics 192, Springer Verlag, New York, 1971, 1-89.

[18] S. Lojasiewicz, Triangulation of semi-analytic sets, Ann. Scuola Norm. Pisa 18 (1964), 449474. MR0173265 (30:3478)

[19] S. Lojasiewiz, Ensembles semi-analytiques (mimeographed notes), I.H.E.S., Bures-sur-Yvette, France, 1965

[20] J. Mather, Stability of $C^{\infty}$ mappings: the division theorem, Ann. Math. 87 (1968), 89-104. MR0232401 (38:726)

[21] J. Mather, Stability of $C^{\infty}$ mappings: infinitesimal stability implies stability, Ann. Math. 89 (1969), 254-291. MR0259953 (41:4582)

[22] J. Mather, Stability of $C^{\infty}$ mappings: finitely determined map-germs, Publ. Math. I.H.E.S. 35 (1969), 127-156.

[23] J. Mather, Stability of $C^{\infty}$ mappings: classification of stable germs by $\mathbb{R}$-algebras, Publ. Math. I.H.E.S. 37 (1970), 223-248. MR0275460 (43:1215b)

[24] J. Mather, Stability of $C^{\infty}$ mappings: transversality, Adv. Math. 4 (1970), 301-335. MR0275461 (43:1215c)

[25] J. Mather, "Stability of $C^{\infty}$ mappings: the nice dimensions", in Proceedings of Liverpool Singularities - Symposium 1, Lecture Notes in Mathematics 192, Springer Verlag, New York (1971), 207-253. MR0293670 (45:2747) 
[26] J. Mather, Notes on Topological Stability, Harvard University, 1970.

[27] J. Mather, "Stratifications and mappings", in Dynamical Systems, Proc. Sympos., Univ. Bahia, Salvador, 1971, pp. 195-232. Academic Press, New York, 1973. MR0368064 (51:4306)

[28] J. Mather, "How to stratify manifolds and jet spaces", in Singularités d'Applications Différentiables, Lecture Notes in Mathematics 135 (1976), Springer Verlag, Berlin, pp. 128176. MR0455018 (56:13259)

[29] M. Pflaum, Analytic and Geometric Study of Stratified Spaces, Lecture Notes in Mathematics 1768, Springer Verlag, 2001. MR1869601 (2002m:58007)

[30] K. Sato, Local triangulation of real analytic varieties, Osaka Math. J. 15 (1963), 109-125. MR0156355 (27:6279)

[31] B. Teissier, Théorèmes de finitude en géometrie analytique, Sem. Bourbaki, 1974.

[32] B. Teissier, Variétés polaires locales et conditions de Whitney, C. R. Acad. Sci. Paris Sér. A-B 290 (1980), A799-A802. MR580569 (82m:32006)

[33] R. Thom, "Local topological properties of differentiable mappings", in Colloquium on Differential Analysis (Tata Institute, Bombay, 1964), Oxford University Press, 1964, pp. 191-202. MR0195102 (33:3307)

[34] R. Thom, La stabilité topologique des applications polynomiales, L'Enseignement Math. (2) 8 (1962), 24-33. MR0148079 (26:5588)

[35] R. Thom, Ensembles et morphismes stratifiés, Bull. Amer. Math. Soc. 75 (1969), no. 2, 240-284. MR0239613(39:970)

[36] R. Thom, "The bifurcation subset of a space of maps", in Manifolds-Amsterdam 1970, Lecture Notes in Mathematics, vol. 197, Springer Verlag, New York, 1971. MR0281222 (43:6941)

[37] A. Verona, Stratified Mappings - Structure and Triangulability, Lecture Notes in Mathematics 1102, Springer Verlag, 1984. MR.771120 (86k:58010)

[38] B. L. van der Waerden, Topologische Begründung des Kalküls der abzählenden Geometrie, Math. Ann. 102 (1929), 337-362.

[39] H. Whitney, On singularities of mappings of euclidean spaces. I. Mappings of the plane into the plane, Ann. of Math. (2) 62 (1955), 374-410. MR0073980 (17:518d)

[40] H. Whitney, Complexes of manifolds, Proc. Nat. Acad. Sci. U.S.A. 33, (1947), 10-11. MR0019306 (8:398a)

[41] H. Whitney, Elementary structure of real algebraic varieties, Ann. Math. 66 (1957), 545-556. MR0095844 (20:2342)

[42] H. Whitney, "Local properties of analytic varieties", in Differential and Combinatorial Topology, Princeton Univ. Press, Princeton, New Jersey, 1965. MR0188486 (32:5924)

[43] H. Whitney, Tangents to an analytic variety. Ann. of Math. (2) 81 (1965), 496-549. MR0192520(33:745)

Institute for Advanced Study, Princeton, New Jersey

E-mail address: goresky@ias.edu 